\title{
Review Article \\ Transcription Regulation of E-Cadherin by Zinc Finger E-Box Binding Homeobox Proteins in Solid Tumors
}

\author{
Thian-Sze Wong, Wei Gao, and Jimmy Yu-Wai Chan \\ Department of Surgery, The University of Hong Kong, Pokfulam, Hong Kong \\ Correspondence should be addressed to Thian-Sze Wong; thiansze@graduate.hku.hk
}

Received 28 February 2014; Revised 13 July 2014; Accepted 28 July 2014; Published 13 August 2014

Academic Editor: Valli De Re

Copyright (C) 2014 Thian-Sze Wong et al. This is an open access article distributed under the Creative Commons Attribution License, which permits unrestricted use, distribution, and reproduction in any medium, provided the original work is properly cited.

Downregulation of E-cadherin in solid tumors with regional migration and systematic metastasis is well recognized. In view of its significance in tumorigenesis and solid cancer progression, studies on the regulatory mechanisms are important for the development of target treatment and prediction of clinical behavior for cancer patients. The vertebrate zinc finger E-box binding homeobox (ZEB) protein family comprises 2 major members: ZEB1 and ZEB2. Both contain the motif for specific binding to multiple enhancer boxes (E-boxes) located within the short-range transcription regulatory regions of the E-cadherin gene. Binding of ZEB1 and ZEB2 to the spaced E-cadherin E-boxes has been implicated in the regulation of E-cadherin expression in multiple human cancers. The widespread functions of ZEB proteins in human malignancies indicate their significance. Given the significance of E-cadherin in the solid tumors, a deeper understanding of the functional role of ZEB proteins in solid tumors could provide insights in the design of target therapy against the migratory nature of solid cancers.

\section{Introduction}

Epithelial cadherin (E-cadherin, cadherin type 1, CD324, or $\mathrm{CDH} 1)$ is involved in the cell cohesiveness and assembly of identical or different cell types during tissue construction and morphogenesis [1]. E-cadherin functions as adhesion molecule at adherens junctions and binds cells through homophilic interactions (i.e., E-cadherin on one cell binds to another E-cadherin molecule on the neighboring cell) in a $\mathrm{Ca}^{2+}$-depending manner. Removal of the calcium ion from the extracellular environment will disrupt the homophilic interactions between E-cadherin molecules, loose the contact between adjacent cells, and promote degradation. Precise transcriptional control of E-cadherin gene expression is essential during developmental reprogramming, cellular differentiation, and cancer progression $[2,3]$. Further, Ecadherin suppression enhances the development of migratory and invasive phenotype by increasing cell motility and facilitating dissociation from the surrounding extracellular matrix of the primary site. Hence, exploiting the fundamental processes involved in E-cadherin suppression is thought to have a significant implication in the context of cancer prevention and migration inhibition.
Destruction of the cadherin-cadherin adhesion linkages at the cell junction is the initial step for cell dissociation and detachment. In solid cancers, cancer cells prompt to change the degree of cell adhesiveness in order to disseminate from the primary cancer site by altering E-cadherin expression [4]. Hereby cancer cells could acquire the mesenchymal phenotype which facilitates them to invade into the surrounding tissues and through basement membranes [5]. This process is referred to epithelial-mesenchymal transition (EMT) and the motile mesenchymal-like cells are characterized by repression of epithelial-associated genes and expression of filopodia and lamellipodia [6]. With the advances in molecular technique, it is now recognized that timely and precise control of E-cadherin expression plays a pivotal role in the molecular reprogramming during EMT and is closely linked with cancer aggressiveness.

\section{Enhancer Box (E-Box) at the Promoter Region of E-Cadherin Encoding Gene}

Transcription factors could bind to the cis-regulatory elements in the promoter region of eukaryotic genes [7]. 
The $5^{\prime}$ proximal promoter regions of E-cadherin gene contain GC-rich sequence, palindromic sequence E-pal, and E-boxes which allows direct binding of specific transcription regulators [8-11]. Although the transcription regulation mechanism of E-cadherin in cancer cells is not fully elucidated, emerging evidence suggested that coordinated recruitment of different transcription factors/repressors to the promoter region plays a key role in controlling timely expression of E-cadherin in different developmental stages. Enhancer box or E-box motifs $\left(5^{\prime}\right.$-CAnnTG- $\left.3^{\prime}\right)$ are palindromic sequence elements which are the binding sites of basic helix-loop-helix (bHLH) class of DNA-binding transcription factors [12, 13]. Using serial $5^{\prime}$ deletion constructs and mutated constructs containing E-cadherin promoter, it has been demonstrated that there are at least 2 E-box elements present in the promoter region with the essential role in controlling E-cadherin expression in both mouse and human genome [11, 14]. Theoretically, the binding of transcription activators or repressors to the E-boxes of the E-cadherin gene could control gene expression at transcription level by allowing the binding of coregulatory proteins.

\section{Zinc Finger E-Box Binding Homeobox (ZEB) Protein Family}

Binding of ZEB1 and ZEB2 to the E-cadherin E-boxes has been implicated in the regulation of E-cadherin expression in multiple human cancers [15]. ZEB proteins are sequencespecific DNA-binding transcription factors. In upper vertebrates, the ZEB belongs to the zfh family comprising ZEB1 (deltaEF1) and ZEB2 (Smad-interacting protein 1, SIP1) [16]. The zhf family members are characterized by the characteristic flanking zinc finger clusters and homeodomainlike domain in their protein with specific DNA-binding ability $[17,18]$. ZEB1 and ZEB2 contain the helix-loop-helix motif allowing them to bind to the bipartite E-boxes within the E-cadherin promoter region with high specificity [3]. Controlled expression of ZEB protein is critical based on the fact that ZEB null mice will die shortly after birth [19]. In normal tissues, expression of ZEB1 and ZEB2 is observed in tissues undergoing differentiation such as $\mathrm{T}$ cell differentiation and skeletal differentiation $[20,21]$. In addition, expression of ZEB proteins is discriminative between cancers with different grading and cancer types [22-25]. ZEB expression is partly controlled by epigenetic mechanisms based on the observation that the transcriptional functions of $\mathrm{ZEB}$ are responsive to the HDAC inhibitor Trichostatin A [26]. By recruiting different coactivators or corepressors, ZEB proteins can perform different functions in the context of chromatin remodeling $[3,27]$. With the recruitment of C-terminal binding protein $\mathrm{CtBP}, \mathrm{ZEB}$ proteins function as transcription repressors [28]. CtBP1 could interact with histone deacetylase to attenuate gene expression by targeting the promoter region [29]. CtBP2 could interact with the ZEB proteins through the three PLDLS-like motifs and mediate transcription suppression [30]. In the presence of CtBP1/2, transcription repression effect was remarkable increased [31]. However, it should be noted that CtBPs binding to
ZEB protein is not always necessary in the ZEB-mediated transcription attenuation [32]. Sumoylation (addition of ubiquitin-like modifier SUMO to the lysine residues) of ZEB protein at Lys391 and Lys866 by the polycomb protein Pc2 could alleviate the E-cadherin repression mediated by ZEB proteins [33]. Recent findings suggested that ZEB expression is controlled by the microRNA which targets the ZEB mRNA transcripts [34-36]. In addition, ZEB could control the microRNA expression by interfering the microRNA promoter activity forming a reciprocal feedback loop in controlling EMT [37]. At present, the mechanism for ZEB to switch from transcription repressors to activators remains poorly understood. In oligodendrocytes, Sip1 can activate Smad7 transcription and modulate various developmental stages [38]. Further, it has been demonstrated that ZEB2 can form complexes with the coactivators p300 and pCAF (p300/CBP associated factor) [39]. Evolutionary functional analysis on vertebrate ZEB protein suggested that the ZEB protein contains functional CtBP-interacting domain, Smadbinding domain, homeodomain, and sumoylation sites which could possibly be the potential sites for its regulation [40].

Dysregulation of ZEB1/2 and E-cadherin has been involved in diverse tumorigenic processes resulting in the development of mesenchymal phenotype, stem-like cell character, resistance to therapeutic agents, aggressiveness during EMT, adaptive stages under hypoxic microenvironment, and cancer progression. Given the significance of E-cadherin in the solid tumors, a deeper understanding of the properties of ZEB proteins is critical. Here, we reviewed the current evidence on transcription regulation of E-cadherin by ZEB1 and ZEB2 proteins in solid tumors.

\section{Bladder and Renal Cancer}

Downregulation of E-cadherin has been implicated in the migration and invasion of bladder cancer cells [41]. In clinical specimens, reduced E-cadherin expression accompanied with increased CD10 (a membrane-bound zinc-dependent metalloprotease) expression is observed in both transitional cell carcinoma and squamous cell carcinoma [42]. In addition, the specific association of E-cadherin reduction with urothelial cell carcinoma leading to the suggestion that loss of E-cadherin is responsible for the progression, invasion, and metastasis of cancer cells derived from the transitional epithelium [42]. In plasmacytoid urothelial carcinoma, complete loss of E-cadherin in the cell membrane is found in more than $70 \%$ and nuclear accumulation is detected in $48 \%$ of the patients [43]. In urothelial carcinoma, E-cadherin level is an indicator of poor prognosis with linking to tumor recurrence and disease-free survival rates [44]. Methylation analysis showing that promoter DNA hypermethylation is a major contributor, which attenuates transcription activity of the Ecadherin gene. With the use of meta-analysis, it is shown that E-cadherin hypermethylation in bladder cancer was prevalent in the Asian populations in comparison with the Caucasian populations [45]. Although membrane E-cadherin is frequently lost in the tumor cells, soluble E-cadherin could be detected in the urine of bladder cancer patients 
and is correlated with the tumor size and lymph node metastasis [46].

ZEB dysregulation is involved in the TGF- $\beta$ 1-induced EMT in renal tubular epithelial cancer cells and is closely associated with the microRNA-200 family [47, 48]. ZEB1 expression is higher in the high-grade urothelial carcinoma in comparison with the low-grade counterparts [24]. In contrast, ZEB2 expression is significantly higher in infiltrating carcinoma than high-grade urothelial carcinoma [24]. It has been suggested that ZEB1 expression is regulated by nuclear $\beta$-catenin upon stimulation [49]. In bladder cancer, $\beta$-catenin signaling cascades can be activated by various routes. In which, many evidence pointed to the glycogen synthase kinase $3 \beta-(\mathrm{GSK} 3 \beta-)$ ZEB1 cascade which was triggered through phosphatidylinositol 3-kinase (PI3 K)/Akt pathways [49-51]. Further, noncoding RNA including microRNA-23b and long noncoding RNA MALAT-1 has also been suggested to be the transcriptional regulator of ZEB1 and ZEB2 in bladder cancers $[52,53]$.

\section{Brain Cancer}

In intracranial cancers, glioblastoma is the most common form [54]. At present, there is still no effective curative treatment for malignant glioblastoma and the survival time is $<1$ year upon diagnosis. The 5 -year survival rate is less than 5\% if the cancer is treated with radiotherapy alone [55]. Although E-cadherin suppression is observed in the brain cancer tissues, the functions of E-cadherin in the tumor cells remain to be verified as another cadherin member, and $\mathrm{N}$ cadherin seems to plays a more significant role in brain cancer aggressiveness. Low E-cadherin expression is found in most of the glioblastoma tissues and is associated with the differentiation status of the glioblastoma $[56,57]$. In comparison with the tumor tissues, E-cadherin expression is rare in the glioblastoma cell lines $[58,59]$. Locking down E-cadherin expression in the E-cadherin expression glioma cells will have a negative impact on cell proliferation and migration [59]. It has been suggested that E-cadherin plays a different role in the glioblastoma tissues (in comparison with the epithelial cancers) based on the observation that Ecadherin expression in the glioblastoma could possibly be associated with the poor clinical outcomes [59]. At present, little is known about the regulatory mechanism of E-cadherin in brain cancer. In medulloblastoma, the methylation frequency of E-cadherin gene was not high (8\%) [60]. In the context of ZEB suppression, binding of ZEB1 to the Ecadherin promoter was dependent on the activation of NF$\kappa \mathrm{B}$ in glioblastoma [61]. In the glioblastoma cell lines, it has been demonstrated that high ZEB2 levels could suppress Ecadherin, thereby regulating cancer cell differentiation [62].

\section{Breast Cancer}

Loss of E-cadherin is characterized in the aggressive breast cancers including aggressive lobular carcinoma and lobular carcinoma in situ in comparison with the less invasive tumor type such as ductal cancers [63]. This led to the suggestion that E-cadherin is involved in mediating tumor progression and metastasis in the breast cancers. Three E-box elements have been suggested to be involved in E-cadherin silencing [9]. It has been shown that the ZEB1 expression is upregulated by steroid hormones such as progesterone [64]. A subpopulation of breast cancer cells with CD44+/CD24- phenotype displays characteristic behavior of stem/progenitor cell and EMT features showing high invasive ability and high expression of ZEB1 and ZEB2 [65]. Naturally occurring agents such as Garcinol (extracts from Garcinia indica) targeting the EMT pathways could function by downregulating ZEB1 and ZEB2 leading to E-cadherin upregulation [66].

\section{Cervical Cancer}

In normal cervix, E-cadherin expression is found on the cell membrane of the basal and parabasal cells [67]. Loss of E-cadherin is linked with the high-risk human papillomaviruses early oncoproteins E5 [68]. Forced expression of E-cadherin in the keratinocyte cell line immortalized with HPV-16 E6 and E7 proteins could reverse the invasive phenotype [69]. The E-cadherin gene is subjected to aberrant DNA hypermethylation and the hypermethylated DNA is detectable in serum of cervical cancer patients with high risk for relapse [70]. E-cadherin expression in cervical cancer could be reactivated using HDAC inhibitor valproic acid (VPA) suggesting that histone modification and chromatin remodeling are involved in the regulation of E-cadherin in cervical cancers [71]. In the widely used cervical cancer model Hela, E-cadherin expression is undetectable [72]. Expression analysis shows that the loss and the resulting migration property are regulated by ZEB1 [73]. Low-dose radiation treatment will suppress E-cadherin expression in cervical cancer cell lines [74]. Although hypoxic has been suggested to be involved in E-cadherin suppression in solid tumors, the oxygenation status (measured by microelectrodes) has no direct correlation with the tumor E-cadherin levels in the squamous cell carcinoma of uterine cervix [75]. At present, whether ZEB1 and ZEB2 involved in the cervical cancers remained to be explored in further details. Clinically, ZEB1 expression was found in over 95\% cervical cancer and the expression level was significantly associated with International Federation of Gynecology and Obstetrics (FIGO) stages and regional lymph node metastasis [67].

\section{Colon Cancer}

The intestinal epithelium has even expression of E-cadherin in the intestinal crypts or surface epithelium [76]. E-cadherin suppression will affect the phenotypic characteristics and physiological state of colon cancer cells by reducing cell-cell adhesiveness [77]. Targeting E-cadherin inhibits glandular differentiation accounting for the undifferentiated phenotype [78]. Poorly differentiated colon cancer cells with E-cadherin expression will have an epithelial-like morphology, elevated $\mathrm{Ca}^{2+}$-dependent cell-cell aggregation, increased cell adhesiveness, and reduced cell motility [79]. In adenocarcinoma, there is an about 2 -fold reduction in the E-cadherin transcript 
level in comparison with the normal colon tissues [80]. Soluble E-cadherin with the 75-85 kDa extracellular domains could be detected in the urine of colon cancer patients [81]. The association between ZEB1 with E-cadherin expression has been reported in the colon cancer cells $[82,83]$.

\section{Endometrial Cancer}

The association between E-cadherin loss and the invasive endometrial cancer is demonstrated by immunohistochemical staining [84]. Loss of E-cadherin has strong association with the histological subtypes of endometrial cancer. The loss is more prevalent in poorly differentiated (International Federation of Gynecology and Obstetrics (FIGO) Grade III) uterine endometrioid adenocarcinomas in comparison with the uterine serous carcinoma [85]. It is suggested that loss of E-cadherin is an early step in endometrioid cancer metastasis and the expression patterns has strong prognostic association with overall morality, disease progression, and extrapelvic recurrence $[84,86]$. The loss is partly linked with E-cadherin gene hypermethylation with higher incidence in the high stage tumor [87]. In the context of ZEB expression, ZEB1 expression is not detected in the normal endometrial epithelium [19]. Exclusive expression of ZEB1 (without ZEB2) is reported in human uterus [88]. ZEB1 expression is altered in the aggressive endometrial cancer including FIGO grade 3 endometrioid adenocarcinomas, uterine serous carcinomas, and malignant mixed Müllerian tumors [89]. In differentiated Ishikawa cell line, increased ZEB1 expression could trigger the development of migratory phenotype [89]. In mouse uterine stroma and myometrium, ZEB1 protein upregulation is partly controlled by estrogen and progesterone. In the estrogen-treated mouse uterus, colocalization of ER and ZEB1 is observed [19]. Based on the expression patterns of ZEB1 in human endometrial biopsies collected at menstrual cycle with high proliferation rate, it is postulated that estrogen and progesterone could control the ZEB1 expression in human myometrial cells [19].

\section{Gastric Cancer}

Alteration of E-cadherin gene expression is common in gastric cancers. Reduced/loss of E-cadherin expression could be caused by promoter hypermethylation induced by the microaerophilic gram-negative bacteria, Helicobacter pylori [90-92]. Helicobacter pylori induced E-cadherin hypermethylation could be reversed if the bacteria are eradicated with antibiotics in the early stages $[92,93]$. Helicobacter pylori can induce the mesenchymal phenotype in gastric epithelial cell lines after $24 \mathrm{~h}$ in contact with elongated phenotype and loosen intercellular junctions [94]. Further, ZEB1 transcripts were increased and the corresponding protein was accumulated in the nucleus when the gastric epithelial cells are in contact with the wild type Helicobacter pylori [94]. ZEB1 expression level was correlated with the mesenchymal phenotype displayed by the gastric cancer [5]. In comparison with other EMT markers including Snail-1 and vimentin, aberrant expression of ZEB1 is more common in gastric cancers [95]. In human gastric cell lines, treatment with ZEB1 siRNA could effectively abrogate the mobility of cancer cells [5]. Strong expression correlation between ZEB2 and Ecadherin mRNA has been demonstrated in gastric carcinoma [96]. Gastric cancer stem cell will express a specific surface marker CD44. CD44 expression is absent in the normal epithelium and the expression will increase when the cancer progress into advanced stages [95]. CD44 expression was correlated with ZEB1 expression and was inversely correlated with the E-cadherin levels in the gastric cancer [95]. Apart from Helicobacter pylori, the nicotine in tobacco could also induce E-cadherin suppression by upregulating ZEB1 through the alpha7 nicotinic acetylcholine receptor in gastric cancer cells [97]. Further, continuous exposure to the lowoxygen environment could also be a contributing factor in ZEB1 and ZEB2 upregulation in gastric cancer [98].

\section{Head and Neck Cancers}

In head and neck cancers, loss of cell-cell adhesion resulting in stromal and vascular invasion as a consequence of Ecadherin dysregulation is well documented [99]. Loss of Ecadherin is common in the tumor borders in comparison with the tumor center [100]. In head and neck cancer cell lines, reduced E-cadherin expression will lead to the loss of epithelioid cell morphology [101]. E-cadherin expression is suppressed in laryngeal carcinoma, especially in supraglottic carcinoma, with significant association to poor differentiation, nodal metastasis, and advanced clinical stages [102,103]. E-cadherin is suggested to be useful in identifying false clinically negative nodes (occult metastases) in laryngeal carcinoma patients [104]. E-cadherin could be suppressed by DNA hypermethylation or the oncoprotein expressed by the human papilloma virus $[105,106]$. In addition, the loss is possibly linked with the inflammation response. Treatment with proinflammatory mediator Interleukin-1 $\beta$ on the head and neck squamous cell carcinoma cell lines will promote ZEB1 binding to the promoter region of E-cadherin [107]. The expression level of ZEB2 is correlated with delayed neck metastasis in stage I/II tongue squamous cell carcinoma patients [108].

Undifferentiated nasopharyngeal carcinoma is a unique head and neck cancer with extremely high sensitivity to ionizing radiation. Hence, radiotherapy is the first line treatment for the primary NPC patients especially when the cancer is still in the early stages. However, it is also noticed that ionizing radiation treatment may promote residual cancer migration and invasion by controlling E-cadherin expression [109]. Cancer cells with low E-cadherin level tend to be resistant to the radiation with higher clonogenic survival rate after exposing to $\gamma$-irradiation [109]. Further, E-cadherin loss is associated with the heterogeneous tumor microenvironment. Under hypoxic condition, E-cadherin expression is suppressed. The suppression was reversible upon oxygenation [109]. Suppressing E-cadherin expression by increasing ZEB1 
expression using AKT inhibitor GSK690693 could enhance the sensitive of nasopharyngeal carcinoma cells to ionizing radiation [110].

\section{Liver and Pancreatic Cancer}

In mouse liver cancer models, loss of E-cadherin will result in metastasis [111]. Downregulation of E-cadherin could induce migration and promote EMT in liver cancer and pancreatic ductal adenocarcinoma $[112,113]$. In human liver cancer, E-cadherin repression is more common in poorly differentiated cases with increased intrahepatic metastasis and poor prognosis [114]. E-cadherin suppression could be induced by the hepatitis $\mathrm{C}$ virus via the induced expression of osteopontin [115]. The tumor suppressing effects of Ecadherin are illustrated in liver-specific E-cadherin knockout mice. E-cadherin knockout mice will develop spontaneous liver cancer and the loss will promote chemical induced (with diethylnitrosamine) liver cancer with strong expression of stem cell marker CD44 and EMT marker vimentin [116]. Upregulation of ZEB1 is associated with thrombomodulin, a cell surface-expressed glycoprotein that is involved in inflammation and thrombosis and Claudin-1, an integral membrane protein $[117,118]$. In addition, the tumor suppressor p53 could suppress ZEB1 and ZEB2 expression in the liver cancer cell lines by controlling their target microRNA expression [119]. Increase in ZEB1 expression is associated with the advanced TNM stages, intrahepatic metastasis, vascular invasion, and frequent early recurrence [120]. The inverse correlation between ZEB1 and E-cadherin has been reported in metastatic liver cancer cell lines and pancreatic tumor cell lines [113]. In pancreatic cancer, E-cadherin suppression is significantly correlated with ZEB1 and ZEB2 expression level and poor prognosis [121].

\section{Lung Cancer}

In lung cancer, genetic mutation of E-cadherin is the primary reason for E-cadherin inactivation [122]. Loss of E-cadherin is associated with the differentiation status and regional lymph node status $[123,124]$. Activation of nuclear factor$\kappa \mathrm{B}(\mathrm{NF}-\kappa \mathrm{B})$ signaling pathways is an important regulation mechanism for E-cadherin expression in lung cancers. In alveolar type II epithelial carcinoma cell line, regulation of E-cadherin expression is partly controlled by Tank-binding kinase-1 (TBK1), inhibitor $\kappa \mathrm{B}(\mathrm{I} \kappa \mathrm{B})$ kinase-related kinase, through activating NF- $\kappa \mathrm{B}$ [125]. Knocking down E-cadherin in non-small cell lung cancer cells will activate the epidermal growth factor receptor (EGFR)-MEK/ERK signaling cascade, which subsequently induce matrix metalloproteinase 2 expressions [126]. Apart from transcription regulation, it has been reported that the non-small cell lung cancer cell aberrantly expressed a misspliced (exon 11) E-cadherin transcript which was rapidly degraded by the nonsense mediated decay pathway [127]. In addition, epigenetic modification of the E-cadherin genes including DNA methylation and histone modification has been implicated in E-cadherin expression. Treatment of lung cancer cells with histone deacetylase inhibitor will inhibit the suppressing function by hindering the binding to the target sequence $[128,129]$. The E-cadherin levels could be restored with the use of HDAC inhibitor Trichostatin A (7-[4-(dimethylamino)phenyl]-N-hydroxy-4,6dimethyl-7-oxohepta-2,4-dienamide) or DNMT inhibitor $5^{\prime}$ Aza-deoxycytidine and the effects are partly linked with the suppression of ZEB1 in the non-small cell lung cancers [130]. ZEB1 could inhibit E-cadherin expression by recruiting histone deacetylases to the promoter regions [131]. ZEB1 upregulation in lung cancer could be controlled by cyclooxygenase-2 [132]. The expression level of E-cadherin and ZEB1 is a useful indicator of cancer cell sensitive to target therapy including epidermal growth factor receptor (EGFR) tyrosine kinase inhibitors, gefitinib, and erlotinib [131]. In addition, ZEB1 is also involved in the radiation-induced epithelial-mesenchymal transition [125]. ZEB1 expression level could also be a predictor to therapeutic responses such as resistance to epidermal growth factor receptor inhibitors for lung cancers [128].

\section{Conclusions}

The exact timing of ZEB1 and ZEB2 upregulation during malignant transformation is not clear yet. It is evidenced that ZEB1 and ZEB2 expression is induced by a sudden changes in the tumor microenvironment such as varying oxygen tensions, exposing to ionizing radiation, contacting with chemotherapeutic agents, and or demethylating agents. In several virus-associated cancers, it was found that ZEB1 and ZEB2 expression is controlled by the viral oncoproteins. In view of the fact that E-cadherin expression could counteract the migratory or invasive property of cancer cells, treatment methods targeting the suppressing mechanisms and triggering the reexpression of E-cadherin are potentially useful in controlling regional and distant metastasis. Hence, molecular dissection of the underlying mechanisms and the pathological consequence of ZEB protein upregulation in Ecadherin suppression will be useful in ameliorating theses effects in the future.

\section{Conflict of Interests}

The authors declare that there is no conflict of interests regarding the publication of this paper.

\section{References}

[1] M. Takeichi, "The cadherins: cell-cell adhesion molecules controlling animal morphogenesis," Development, vol. 102, no. 4, pp. 639-655, 1988.

[2] M. Takeichi, "Cadherin cell adhesion receptors as a morphogenetic regulator," Science, vol. 251, no. 5000, pp. 1451-1455, 1991.

[3] H. Peinado, D. Olmeda, and A. Cano, "Snail, ZEB and bHLH factors in tumour progression: an alliance against the epithelial phenotype?" Nature Reviews Cancer, vol. 7, no. 6, pp. 415-428, 2007.

[4] J. P. Thiery, H. Acloque, R. Y. J. Huang, and M. A. Nieto, "Epithelial-mesenchymal transitions in development and disease," Cell, vol. 139, no. 5, pp. 871-890, 2009. 
[5] T. Murai, S. Yamada, B. C. Fuchs et al., "Epithelial-tomesenchymal transition predicts prognosis in clinical gastric cancer," Journal of Surgical Oncology, vol. 109, no. 7, pp. 684689, 2014.

[6] R. Kalluri and E. G. Neilson, "Epithelial-mesenchymal transition and its implications for fibrosis," Journal of Clinical Investigation, vol. 112, no. 12, pp. 1776-1784, 2003.

[7] M. Bensimhon, J. Gabarro-Arpa, R. Ehrlich, and C. Reiss, "Physical characteristics in eucaryotic promoters," Nucleic Acids Research, vol. 11, no. 13, pp. 4521-4540, 1983.

[8] A. Cano, M. A. Pérez-Moreno, I. Rodrigo et al., "The transcription factor Snail controls epithelial-mesenchymal transitions by repressing E-cadherin expression," Nature Cell Biology, vol. 2, no. 2, pp. 76-83, 2000.

[9] K. M. Hajra and E. R. Fearon, "The SLUG zinc-finger protein represses E-cadherin in breast cancer," Cancer Research, vol. 62, no. 6, pp. 1613-1618, 2002.

[10] J. Behrens, O. Löwrick, L. Klein-Hitpass, and W. Birchmeier, "The E-cadherin promoter: functional analysis of a G.C-rich region and an epithelial cell-specific palindromic regulatory element," Proceedings of the National Academy of Sciences of the United States of America, vol. 88, no. 24, pp. 11495-11499, 1991.

[11] L. A. Giroldi, P. Bringuier, M. de Weijert, C. Jansen, A. van Bokhoven, and J. A. Schalken, "Role of E boxes in the repression of E-cadherin expression," Biochemical and Biophysical Research Communications, vol. 241, no. 2, pp. 453-458, 1997.

[12] J. Weinberger, D. Baltimore, and P. A. Sharp, "Distinct factors bind to apparently homolgous sequences in the immunoglobulin heavy-chain enhancer," Nature, vol. 322, no. 6082, pp. 846$848,1986$.

[13] M. E. Massari and C. Murre, "Helix-loop-helix proteins: regulators of transcription in eucaryotic organisms," Molecular and Cellular Biology, vol. 20, no. 2, pp. 429-440, 2000.

[14] J. Behrens, O. Lowrick, L. Klein-Hitpass, and W. Birchmeier, "The E-cadherin promoter: functional analysis of a G.C-rich region and an epithelial cell-specific palindromic regulatory element," Proceedings of the National Academy of Sciences of the United States of America, vol. 88, no. 24, pp. 11495-11499, 1991.

[15] J. Comijn, G. Berx, P. Vermassen et al., "The two-handed E box binding zinc finger protein SIP1 downregulates E-cadherin and induces invasion," Molecular Cell, vol. 7, no. 6, pp. 1267-1278, 2001.

[16] R. Sekido, K. Murai, J. Funahashi et al., "The $\delta$-crystallin enhancer-binding protein $\delta \mathrm{EF} 1$ is a repressor of E2- boxmediated gene activation," Molecular and Cellular Biology, vol. 14, no. 9, pp. 5692-5700, 1994.

[17] H. Yasuda, A. Mizuno, T. Tamaoki, and T. Morinaga, "ATBF1, a multiple-homeodomain zinc finger protein, selectively downregulates AT-rich elements of the human $\alpha$-fetoprotein gene," Molecular and Cellular Biology, vol. 14, no. 2, pp. 1395-1401, 1994.

[18] T. Genetta, D. Ruezinsky, and T. Kadesch, "Displacement of an E-box-binding repressor by basic helix-loop-helix proteins: implications for B-cell specificity of the immunoglobulin heavy-chain enhancer," Molecular and Cellular Biology, vol. 14, no. 9, pp. 6153-6163, 1994.

[19] N. S. Spoelstra, N. G. Manning, Y. Higashi et al., "The transcription factor ZEB1 is aberrantly expressed in aggressive uterine cancers," Cancer Research, vol. 66, no. 7, pp. 3893-3902, 2006.

[20] Y. Higashi, H. Moribe, T. Takagi et al., "Impairment of T cell development in $\delta \mathrm{EF} 1$ mutant mice," The Journal of Experimental Medicine, vol. 185, no. 8, pp. 1467-1479, 1997.
[21] T. Takagi, H. Moribe, H. Kondoh, and Y. Higashi, "DeltaEF1, a zinc finger and homeodomain transcription factor, is required for skeleton patterning in multiple lineages," Development, vol. 125, no. 1, pp. 21-31, 1998.

[22] A. A. Postigo and D. C. Dean, "Differential expression and function of members of the zfh-1 family of zinc finger/homeodomain repressors," Proceedings of the National Academy of Sciences of the United States of America, vol. 97, no. 12, pp. 6391-6396, 2000.

[23] J. E. Remacle, H. Kraft, W. Lerchner et al., "New mode of DNA binding of multi-zinc finger transcription factors: $\delta \mathrm{EF} 1$ family members bind with two hands to two target sites," The EMBO Journal, vol. 18, no. 18, pp. 5073-5084, 1999.

[24] H. Lee, S. Y. Jun, Y. S. Lee, H. J. Lee, W. S. Lee, and C. S. Park, "Expression of miRNAs and ZEB1 and ZEB2 correlates with histopathological grade in papillary urothelial tumors of the urinary bladder," Virchows Archiv, vol. 464, no. 2, pp. 213-220, 2014.

[25] E. Oztas, M. E. Avci, A. Ozcan, A. E. Sayan, E. Tulchinsky, and T. Yagci, "Novel monoclonal antibodies detect Smad-interacting protein 1 (SIP1) in the cytoplasm of human cells from multiple tumor tissue arrays," Experimental and Molecular Pathology, vol. 89 , no. 2, pp. 182-189, 2010.

[26] L. A. van Grunsven, V. Taelman, C. Michiels et al., "XSip1 neuralizing activity involves the co-repressor CtBP and occurs through BMP dependent and independent mechanisms," Developmental Biology, vol. 306, no. 1, pp. 34-49, 2007.

[27] G. Verstappen, L. A. Van Grunsven, C. Michiels et al., "Atypical Mowat-Wilson patient confirms the importance of the novel association between ZFHX1B/SIP1 and NuRD corepressor complex," Human Molecular Genetics, vol. 17, no. 8, pp. 1175-1183, 2008.

[28] A. A. Postigo and D. C. Dean, "ZEB represses transcription through interaction with the corepressor CtBP," Proceedings of the National Academy of Sciences of the United States of America, vol. 96, no. 12, pp. 6683-6688, 1999.

[29] A. Sundqvist, K. Sollerbrant, and C. Svensson, "The carboxyterminal region of adenovirus E1A activates transcription through targeting of a C-terminal binding protein-histone deacetylase complex," FEBS Letters, vol. 429, no. 2, pp. 183-188, 1998.

[30] L. Zhao, M. Kuppuswamy, S. Vijayalingam, and G. Chinnadurai, "Interaction of ZEB and histone deacetylase with the PLDLSbinding cleft region of monomeric C-terminal binding protein 2," BMC Molecular Biology, vol. 10, article 89, 2009.

[31] T. Furusawa, H. Moribe, H. Kondoh, and Y. Higashi, "Identification of CtBP1 and CtBP2 as corepressors of zinc fingerhomeodomain factor $\delta \mathrm{EF} 1$, " Molecular and Cellular Biology, vol. 19, no. 12, pp. 8581-8590, 1999.

[32] L. A. van Grunsven, C. Michiels, T. van de Putte et al., "Interaction between Smad-interacting protein-1 and the corepressor C-terminal binding protein is dispensable for transcriptional repression of E-cadherin," Journal of Biological Chemistry, vol. 278, no. 28, pp. 26135-26145, 2003.

[33] J. Long, D. Zuo, and M. Park, "Pc2-mediated sumoylation of Smad-interacting protein 1 attenuates transcriptional repression of E-cadherin," The Journal of Biological Chemistry, vol. 280, no. 42, pp. 35477-35489, 2005.

[34] X. Wang, X. He, F. Zhao et al., "Regulation gene expression of miR200c and ZEB1 positively enhances effect of tumor vaccine B16F10/GPI-IL-21 on inhibition of melanoma growth 
and metastasis," Journal of Translational Medicine, vol. 12, article 68, 2014.

[35] L. Bojmar, E. Karlsson, S. Ellegård et al., "The role of microRNA200 in progression of human colorectal and breast cancer," PLoS ONE, vol. 8, no. 12, article e84815, 2013.

[36] L. Hill, G. Browne, and E. Tulchinsky, "ZEB/miR-200 feedback loop: at the crossroads of signal transduction in cancer," International Journal of Cancer, vol. 132, no. 4, pp. 745-754, 2012.

[37] S. Brabletz and T. Brabletz, "The ZEB/miR-200 feedback loop-a motor of cellular plasticity in development and cancer?" EMBO Reports, vol. 11, no. 9, pp. 670-677, 2010.

[38] Q. Weng, Y. Chen, H. Wang et al., "Dual-mode modulation of Smad signaling by Smad-interacting protein Sip1 is required for myelination in the central nervous system," Neuron, vol. 73, no. 4, pp. 713-728, 2012.

[39] L. A. van Grunsven, V. Taelman, C. Michiels, K. Opdecamp, D. Huylebroeck, and E. J. Bellefroid, " $\delta \mathrm{EF} 1$ and SIP1 are differentially expressed and have overlapping activities during Xenopus embryogenesis," Developmental Dynamics, vol. 235, no. 6, pp. 1491-1500, 2006.

[40] A. Gheldof, P. Hulpiau, F. van Roy, B. de Craene, and G. Berx, "Evolutionary functional analysis and molecular regulation of the ZEB transcription factors," Cellular and Molecular Life Sciences, vol. 69, no. 15, pp. 2527-2541, 2012.

[41] Du. HF, Ou. LP, X. Yang et al., "A new PKC $\alpha / \beta / \mathrm{TBX} 3 / \mathrm{E}-$ cadherin pathway is involved in PLCE-regulated invasion and migration in human bladder cancer cells," Cellular Signalling, vol. 26, no. 3, pp. 580-593, 2014.

[42] O. M. Omran, "Cd10 and e-cad expression in urinary bladder urothelial and squamous cell carcinoma," Journal of Environmental Pathology, Toxicology and Oncology, vol. 31, no. 3, pp. 203-212, 2012.

[43] B. Keck, S. Wach, F. Kunath et al., "Nuclear E-cadherin expression is associated with the loss of membranous E-cadherin, plasmacytoid differentiation and reduced overall survival in urothelial carcinoma of the bladder," Annals of Surgical Oncology, vol. 20, no. 7, pp. 2440-2445, 2013.

[44] S. T. dos Reis, K. R. M. Leite, A. Mosconi Neto et al., "Immune expression of E-cadherin and $\alpha, \beta$ and $\gamma$-catenin adhesion molecules and prognosis for upper urinary tract urothelial carcinomas," International Brazilian Journal of Urology, vol. 38, no. 4, pp. 466-473, 2012.

[45] G. Li, Y. Liu, H. Yin et al., "E-cadherin gene promoter hypermethylation may contribute to the risk of bladder cancer among Asian populations," Gene, vol. 534, no. 1, pp. 48-53, 2014.

[46] R. H. M. Salama, T. H. Selem, M. El-Gammal, A. A. Elhagagy, and S. M. Bakar, "Urinary tumor markers could predict survival in bladder carcinoma," Indian Journal of Clinical Biochemistry, vol. 28, no. 3, pp. 265-271, 2013.

[47] M. Xiong, L. Jiang, Y. Zhou et al., "The miR-200 family regulates TGF- $\beta 1$-induced renal tubular epithelial to mesenchymal transition through smad pathway by targeting ZEB1 and ZEB2 expression," The American Journal of Physiology-Renal Physiology, vol. 302, no. 3, pp. F369-F379, 2012.

[48] S. Oba, S. Kumano, E. Suzuki et al., "miR-200b precursor can ameliorate renal tubulointerstitial fibrosis," PLoS ONE, vol. 5, no. 10, Article ID e13614, 2010.

[49] K. Wu, Z. Ning, J. Zeng et al., "Silibinin inhibits $\beta$-catenin/ZEB1 signaling and suppresses bladder cancer metastasis via dualblocking epithelial-mesenchymal transition and stemness," Cell Signal, vol. 25, no. 12, pp. 2625-2633, 2013.
[50] K. Wu, J. Fan, L. Zhang et al., "PI3K/Akt to GSK3 $\beta / \beta$-catenin signaling cascade coordinates cell colonization for bladder cancer bone metastasis through regulating ZEB1 transcription," Cellular Signalling, vol. 24, no. 12, pp. 2273-2282, 2012.

[51] Y. Matsui, K. Assi, O. Ogawa et al., "The importance of integrinlinked kinase in the regulation of bladder cancer invasion," International Journal of Cancer, vol. 130, no. 3, pp. 521-531, 2012.

[52] S. Majid, A. A. Dar, S. Saini et al., "MicroRNA-23b functions as a tumor suppressor by regulating Zebl in bladder cancer," PLoS ONE, vol. 8, no. 7, Article ID e67686, 2013.

[53] L. Ying, Q. Chen, Y. Wang, Z. Zhou, Y. Huang, and F. Qiu, "Upregulated MALAT-1 contributes to bladder cancer cell migration by inducing epithelial-to-mesenchymal transition," Molecular BioSystems, vol. 8, no. 9, pp. 2289-2294, 2012.

[54] K. Iwami, A. Natsume, and T. Wakabayashi, "Cytokine networks in glioma," Neurosurgical Review, vol. 34, no. 3, pp. 253-263, 2011.

[55] R. Stupp, W. P. Mason, M. J. van den Bent et al., "Radiotherapy plus concomitant and adjuvant temozolomide for glioblastoma," The New England Journal of Medicine, vol. 352, no. 10, pp. 987-996, 2005.

[56] L. Yang, M. Liu, C. Deng, Z. Gu, and Y. Gao, "Expression of transforming growth factor- $\beta 1$ (TGF- $\beta 1$ ) and E-cadherin in glioma," Tumor Biology, vol. 33, no. 5, pp. 1477-1484, 2012.

[57] W. Wu, Y. Tian, H. Wan et al., "Expression of $\beta$-catenin and Eand N-cadherin in human brainstem gliomas and clinicopathological correlations," International Journal of Neuroscience, vol. 123, no. 5, pp. 318-323, 2013.

[58] C. Perego, C. Vanoni, S. Massari et al., "Invasive behaviour of glioblastoma cell lines is associated with altered organisation of the cadherin-catenin adhesion system," Journal of Cell Science, vol. 115, no. 16, pp. 3331-3340, 2002.

[59] L. J. Lewis-Tuffin, F. Rodriguez, C. Giannini et al., "Misregulated E-Cadherin expression associated with an aggressive brain tumor phenotype," PLoS ONE, vol. 5, no. 10, Article ID e13665, 2010.

[60] M. Ebinger, L. Senf, O. Wachowski, and W. Scheurlen, "Promoter methylation pattern of caspase-8, P16INK4A, MGMT, TIMP-3, and E-cadherin in medulloblastoma," Pathology and Oncology Research, vol. 10, no. 1, pp. 17-21, 2004.

[61] L. A. Edwards, K. Woolard, M. J. Son et al., "Effect of brainand tumor-derived connective tissue growth factor on glioma invasion," Journal of the National Cancer Institute, vol. 103, no. 15, pp. 1162-1178, 2011.

[62] S. Qi, Y. Song, Y. Peng et al., “ZEB2 mediates multiple pathways regulating cell proliferation, migration, invasion, and apoptosis in glioma," PLoS ONE, vol. 7, no. 6, Article ID e38842, 2012.

[63] D. Sarrió, B. Pérez-Mies, D. Hardisson et al., "Cytoplasmic localization of p120ctn and E-cadherin loss characterize lobular breast carcinoma from preinvasive to metastatic lesions," Oncogene, vol. 23, no. 19, pp. 3272-3283, 2004.

[64] J. K. Richer, B. M. Jacobsen, N. G. Manning, M. G. Abel, D. M. Wolf, and K. B. Horwitz, "Differential gene regulation by the two progesterone receptor isoforms in human breast cancer cells," Journal of Biological Chemistry, vol. 277, no. 7, pp. 52095218, 2002.

[65] P. Bhat-Nakshatri, H. Appaiah, C. Ballas et al., "SLUG/SNAI2 and Tumor Necrosis Factor Generate Breast Cells With CD44+/CD24- Phenotype," BMC Cancer, vol. 10, article 411, 2010. 
[66] A. Ahmad, S. H. Sarkar, B. Bitar et al., "Garcinol regulates EMT and Wnt signaling pathways in vitro and in vivo, leading to anticancer activity against breast cancer cells," Molecular Cancer Therapeutics, vol. 11, no. 10, pp. 2193-2201, 2012.

[67] C. J. Vessey, J. Wilding, N. Folarin et al., "Altered expression and function of E-cadherin in cervical intraepithelial neoplasia and invasive squamous cell carcinoma," Journal of Pathology, vol. 176, no. 2, pp. 151-159, 1995.

[68] S. Liao, D. Deng, W. Zhang et al., "Human papillomavirus 16/18 E5 promotes cervical cancer cell proliferation, migration and invasion in vitro and accelerates tumor growth in vivo," Oncology Reports, vol. 29, no. 1, pp. 95-102, 2013.

[69] J. Wilding, K. H. Vousden, W. P. Soutter, P. D. McCrea, R. Del Buono, and M. Pignatelli, "E-cadherin transfection downregulates the epidermal growth factor receptor and reverses the invasive phenotype of human papilloma virus- transfected keratinocytes," Cancer Research, vol. 56, no. 22, pp. 5285-5292, 1996.

[70] A. Widschwendter, L. Ivarsson, A. Blassnig et al., "CDH1 AND $\mathrm{CDH} 13$ methylation in serum is an independent prognostic marker in cervical cancer patients," International Journal of Cancer, vol. 109, no. 2, pp. 163-166, 2004.

[71] D. Feng, J. Wu, Y. Tian et al., “ Targeting of histone deacetylases to reactivate tumour suppressor genes and its therapeutic potential in a human cervical cancer xenograft model," PLoS ONE, vol. 8, no. 11, article e80657, 2013.

[72] C. Cunniffe, F. Ryan, H. Lambkin, and B. Brankin, "Expression of tight and adherens junction proteins in cervical neoplasia," British Journal of Biomedical Science, vol. 69, no. 4, pp. 147-153, 2012.

[73] C. Tai, C. Cheng, H. Su et al., “Thrombomodulin mediates the migration of cervical cancer cells through the regulation of epithelial-mesenchymal transition biomarkers," Tumor Biology, vol. 35, no. 1, pp. 47-54, 2014.

[74] S. Yan, Y. Wang, Q. Yang et al., "Low-dose radiation-induced epithelial-mesenchymal transition through NF- $\kappa \mathrm{B}$ in cervical cancer cells," International Journal of Oncology, vol. 42, no. 5, pp. 1801-1806, 2013.

[75] A. Mayer, M. Höckel, N. Schlischewsky, H. Schmidberger, L. C. Horn, and P. Vaupel, "Lacking hypoxia-mediated downregulation of E-cadherin in cancers of the uterine cervix," British Journal of Cancer, vol. 108, no. 2, pp. 402-408, 2013.

[76] A. Doğan, Z. D. Wang, and J. Spencer, "E-cadherin expression in intestinal epithelium," Journal of Clinical Pathology, vol. 48, no. 2, pp. 143-146, 1995.

[77] M. J. Wheelock, "Catenin association with E-cadherin changes with the state of polarity of HT-29 cells," Experimental Cell Research, vol. 191, no. 2, pp. 186-193, 1990.

[78] M. Pignatelli, D. Liu, M. M. Nasim, G. W. H. Stamp, S. Hirano, and M. Takeichi, "Morphoregulatory activities of E-cadherin and beta-1 integrins in colorectal tumour cells," British Journal of Cancer, vol. 66, no. 4, pp. 629-634, 1992.

[79] E. Breen, G. Steele Jr., and A. M. Mercurio, "Role of the Ecadherin $/ \alpha$-catenin complex in modulating cell-cell and cellmatrix adhesive properties of invasive colon carcinoma cells," Annals of Surgical Oncology, vol. 2, no. 5, pp. 378-385, 1995.

[80] S. B. Munro, I. M. Turner, R. Farookhi, O. W. Blaschuk, and S. Jothy, "E-cadherin and OB-cadherin mRNA levels in normal human colon and colon carcinoma," Experimental and Molecular Pathology, vol. 62, no. 2, pp. 118-122, 1995.

[81] M. Katayama, S. Hirai, M. Yasumoto et al., "Soluble fragments of E-cadherin cell adhesion molecule increase in urinary excretion of cancer patients, potentially indicating its shedding from epithelial tumor cells," International Journal of Oncology, vol. 5, no. 5, pp. 1049-1057, 1994.

[82] A. R. Paek, C. H. Lee, and H. J. You, "A role of zinc-finger protein 143 for cancer cell migration and invasion through ZEB1 and Ecadherin in colon cancer cells," Molecular Carcinogenesis, vol. 53, no. S1, pp. E161-E168, 2014.

[83] A. B. Singh, A. Sharma, J. J. Smith et al., "Claudin-1 up-regulates the repressor ZEB-1 to inhibit E-cadherin expression in colon cancer cells," Gastroenterology, vol. 141, no. 6, pp. 2140-2153, 2011.

[84] L. K. Mell, J. J. Meyer, M. Tretiakova et al., "Prognostic significance of E-cadherin protein expression in pathological stage I-III endometrial cancer," Clinical Cancer Research, vol. 10, no. 16, pp. 5546-5553, 2004.

[85] P. W. Schlosshauer, L. H. Ellenson, and R. A. Soslow, “ $\beta$-catenin and E-cadherin expression patterns in high-grade endometrial carcinoma are associated with histological subtype," Modern Pathology, vol. 15, no. 10, pp. 1032-1037, 2002.

[86] Y. T. Kim, E. K. Choi, J. W. Kim, D. K. Kim, S. H. Kim, and W. I. Yang, "Expression of E-cadherin and $\alpha-, \beta-, \gamma$-catenin proteins in endometrial carcinoma," Yonsei Medical Journal, vol. 43, no. 6, pp. 701-711, 2002.

[87] J. H. Park, B. I. Lee, E. S. Song, S. O. Whang, W. Y. Lee, and S. J. Cho, "Hypermethylation of E-cadherin in endometrial carcinoma," Journal of Gynecologic Oncology, vol. 19, no. 4, pp. 241-245, 2008.

[88] A. A. Postigo, "Opposing functions of ZEB proteins in the regulation of the TGF $\beta / \mathrm{BMP}$ signaling pathway," The EMBO Journal, vol. 22, no. 10, pp. 2443-2452, 2003.

[89] M. Singh, N. S. Spoelstra, A. Jean et al., "ZEB1 expression in type I vs type II endometrial cancers: a marker of aggressive disease," Modern Pathology, vol. 21, no. 7, pp. 912-923, 2008.

[90] P. Carneiro, J. Figueiredo, R. Bordeira-Carriço et al., “Therapeutic targets associated to E-cadherin dysfunction in gastric cancer," Expert Opinion on Therapeutic Targets, vol. 17, no. 10, pp. 1187-201, 2013.

[91] Y. Qu, S. Dang, and P. Hou, "Gene methylation in gastric cancer," Clinica Chimica Acta, vol. 424, pp. 53-65, 2013.

[92] G. Carrasco and A. H. Corvalan, "Helicobacter pylori-induced chronic gastritis and assessing risks for gastric cancer," Gastroenterology Research and Practice, vol. 2013, Article ID 393015, 8 pages, 2013.

[93] A. O. O. Chan and A. Rashid, "CpG island methylation in precursors of gastrointestinal malignancies," Current Molecular Medicine, vol. 6, no. 4, pp. 401-408, 2006.

[94] J. Baud, C. Varon, S. Chabas, L. Chambonnier, F. Darfeuille, and C. Staedel, "Helicobacter pylori initiates a mesenchymal transition through ZEB1 in gastric epithelial cells," PLoS ONE, vol. 8, no. 4, Article ID e60315, 2013.

[95] H. S. Ryu, J. Park do, H. H. Kim, W. H. Kim, and H. S. Lee, "Combination of epithelial-mesenchymal transition and cancer stem cell-like phenotypes has independent prognostic value in gastric cancer," Human Pathology, vol. 43, no. 4, pp. 520-528, 2012.

[96] J. Kurashige, H. Kamohara, M. Watanabe et al., "MicroRNA$200 \mathrm{~b}$ regulates cell proliferation, invasion, and migration by directly targeting ZEB2 in gastric carcinoma," Annals of Surgical Oncology, vol. 19, no. 3, pp. S656-S664, 2012.

[97] Y. Lien, W. Wang, L. Kuo et al., "Nicotine promotes cell migration through alpha7 nicotinic acetylcholine receptor in 
gastric cancer cells," Annals of Surgical Oncology, vol. 18, no. 9, pp. 2671-2679, 2011.

[98] Y. Kato, M. Yashiro, S. Noda et al., "Establishment and characterization of a new hypoxia-resistant cancer cell line, OCUM12/Hypo, derived from a scirrhous gastric carcinoma," British Journal of Cancer, vol. 102, no. 5, pp. 898-907, 2010.

[99] V. Matijssen, H. M. Peters, L. Schalkwijk et al., "E-cadherin expression in head and neck squamous-cell carcinoma is associated with clinical outcome," International Journal of Cancer, vol. 55, no. 4, pp. 580-585, 1993.

[100] J. G. Eriksen, T. Steiniche, H. Søgaard, and J. Overgaard, "Expression of integrins and E-cadherin in squamous cell carcinomas of the head and neck," APMIS, vol. 112, no. 9, pp. 560-568, 2004.

[101] A. M. Tomson, J. Scholma, B. Meijer, J. G. J. Koning, K. M. D. de Jong, and M. van der Werf, "Adhesion properties, intermediate filaments and malignant behaviour of head and neck squamous cell carcinoma cells in vitro," Clinical and Experimental Metastasis, vol. 14, no. 6, pp. 501-511, 1996.

[102] E. Zvrko, A. Mikić, and S. Jancić, "Relationship of E-cadherin with cervical lymph node metastasis in laryngeal cancer," Collegium Antropologicum, vol. 36, Supplement 2, pp. 119-124, 2012.

[103] E. Mittari, A. Charalabopoulos, A. Batistatou, and K. Charalabopoulos, "The role of E-cadherin/catenin complex in laryngeal cancer," Experimental Oncology, vol. 27, no. 4, pp. 257-261, 2005.

[104] A. Franchi, O. Gallo, V. Boddi, and M. Santucci, "Prediction of occult neck metastases in laryngeal carcinoma: Role of proliferating cell nuclear antigen, MIB-1, and E-cadherin immunohistochemical determination," Clinical Cancer Research, vol. 2, no. 10, pp. 1801-1808, 1996.

[105] C. J. Marsit, M. R. Posner, M. D. McClean, and K. T. Kelsey, "Hypermethylation of E-cadherin is an independent predictor of improved survival in head and neck squamous cell carcinoma," Cancer, vol. 113, no. 7, pp. 1566-1571, 2008.

[106] A. Al Moustafa, W. D. Foulkes, N. Benlimame et al., "E6/E7 proteins of HPV type 16 and ErbB-2 cooperate to induce neoplastic transformation of primary normal oral epithelial cells," Oncogene, vol. 23, no. 2, pp. 350-358, 2004.

[107] M. Dohadwala, G. Wang, E. Heinrich et al., "The role of ZEB1 in the inflammation-induced promotion of EMT in HNSCC," Otolaryngology-Head and Neck Surgery, vol. 142, no. 5, pp. 753-759, 2010.

[108] K. Sakamoto, Y. Imanishi, T. Tomita et al., "Overexpression of SIP1 and downregulation of e-cadherin predict delayed neck metastasis in stage I/II oral tongue squamous cell carcinoma after partial glossectomy," Annals of Surgical Oncology, vol. 19, no. 2, pp. 612-619, 2012.

[109] Y. Zhou, J. Liu, J. Li et al., "Ionizing radiation promotes migration and invasion of cancer cells through transforming growth factor-beta-mediated epithelial-mesenchymal transition," International Journal of Radiation Oncology Biology Physics, vol. 81, no. 5, pp. 1530-1537, 2011.

[110] W. Chen, S. Wu, G. Zhang, W. Wang, and Y. Shi, "Effect of AKT inhibition on epithelial-mesenchymal transition and ZEB1-potentiated radiotherapy in nasopharyngeal carcinoma," Oncology Letters, vol. 6, no. 5, pp. 1234-1240, 2013.

[111] W. Ding, H. You, H. Dang et al., "Epithelial-to-mesenchymal transition of murine liver tumor cells promotes invasion," Hepatology, vol. 52, no. 3, pp. 945-953, 2010.
[112] T. Liu, Y. Jan, B. Ko et al., "14-3-3e overexpression contributes to epithelial-mesenchymal transition of hepatocellular carcinoma," PLoS ONE, vol. 8, no. 3, Article ID e57968, 2013.

[113] T. Grosse-Steffen, T. Giese, N. Giese et al., "Epithelial-tomesenchymal transition in pancreatic ductal adenocarcinoma and pancreatic tumor cell lines: the role of neutrophils and neutrophil-derived elastase," Clinical and Developmental Immunology, vol. 2012, Article ID 720768, 12 pages, 2012.

[114] M. Hashiguchi, S. Ueno, M. Sakoda et al., "Clinical implication of ZEB-1 and E-cadherin expression in hepatocellular carcinoma (HCC)," BMC Cancer, vol. 13, article 572, 2013.

[115] J. Iqbal, S. McRae, T. Mai, K. Banaudha, M. Sarkar-Dutta, and G. Waris, "Role of hepatitis C virus induced osteopontin in epithelial to mesenchymal transition, migration and invasion of hepatocytes," PLoS One, vol. 9, no. 1, Article ID e87464, 2014.

[116] H. Nakagawa, Y. Hikiba, Y. Hirata et al., "Loss of liver Ecadherin induces sclerosing cholangitis and promotes carcinogenesis," Proceedings of the National Academy of Sciences of the United States of America, vol. 111, no. 3, pp. 1090-1095, 2014.

[117] M. Huang, P. Wei, J. Liu et al., "Knockdown of thrombomodulin enhances HCC cell migration through increase of ZEB1 and decrease of E-cadherin gene expression," Annals of Surgical Oncology, vol. 17, no. 12, pp. 3379-3385, 2010.

[118] Y. Suh, C. Yoon, R. Kim et al., "Claudin-1 induces epithelialmesenchymal transition through activation of the c-Abl-ERK signaling pathway in human liver cells," Oncogene, vol. 32, no. 41, pp. 4873-4882, 2013.

[119] T. Kim, A. Veronese, F. Pichiorri et al., "p53 regulates epithelialmesenchymal transition through microRNAs targeting ZEB1 and ZEB2," Journal of Experimental Medicine, vol. 208, no. 5, pp. 875-883, 2011.

[120] Y. M. Zhou, L. Cao, B. Li et al., "Clinicopathological significance of ZEB1 protein in patients with hepatocellular carcinoma," Annals of Surgical Oncology, vol. 19, no. 5, pp. 1700-1706, 2012.

[121] H. Kurahara, S. Takao, K. Maemura et al., "Epithelialmesenchymal transition and mesenchymal-epithelial transition via regulation of ZEB-1 and ZEB-2 expression in pancreatic cancer," Journal of Surgical Oncology, vol. 105, no. 7, pp. 655-661, 2012.

[122] Q. Fei, H. Zhang, X. Chen et al., "Defected expression of Ecadherin in non-small cell lung cancer," Lung Cancer, vol. 37, no. 2, pp. 147-152, 2002.

[123] N. H. Myong, "Reduced expression of E-cadherin in human non-small cell lung carcinoma," Cancer Treatment and Research, vol. 36, no. 1, pp. 56-61, 2004.

[124] A. Kalogeraki, D. Bouros, O. Zoras et al., "E-cadherin expression on fine-needle aspiration biopsies in primary lung adenocarcinomas is related to tumor differentiation and invasion," Anticancer Research, vol. 23, no. 4, pp. 3367-3371, 2003.

[125] W. Liu, Y. J. Huang, C. Liu et al., "Inhibition of TBK1 attenuates radiation-induced epithelial-mesenchymal transition of A549 human lung cancer cells via activation of GSK-3 $\beta$ and repression of ZEB1," Laboratory Investigation, vol. 94, no. 4, pp. 362370, 2014.

[126] G. Y. Bae, S. J. Choi, J. S. Lee et al., "Loss of E-cadherin activates EGFR-MEK/ERK signaling, which promotes invasion via the ZEB1/MMP2 axis in non-small cell lung cancer," Oncotarget, vol. 4, no. 12, pp. 2512-2522, 2013.

[127] W. Liao, G. Jordaan, M. K. Srivastava, S. Dubinett, and S. Sharma, "Effect of epigenetic histone modifications on Ecadherin splicing and expression in lung cancer," American Journal of Cancer Research, vol. 3, no. 4, pp. 374-389, 2013. 
[128] J. Clarhaut, R. M. Gemmill, V. A. Potiron et al., "ZEB-1, a repressor of the semaphorin $3 \mathrm{~F}$ tumor suppressor gene in lung cancer cells," Neoplasia, vol. 11, no. 2, pp. 157-166, 2009.

[129] M. Kakihana, T. Ohira, D. Chan et al., "Induction of E-cadherin in lung cancer and interaction with growth suppression by histone deacetylase inhibition," Journal of Thoracic Oncology, vol. 4, no. 12, pp. 1455-1465, 2009.

[130] S. Mateen, K. Raina, C. Agarwal, D. Chan, and R. Agarwal, "Silibinin synergizes with histone deacetylase and DNA methyltransferase inhibitors in upregulating e-cadherin expression together with inhibition of migration and invasion of human non-small cell lung cancer cells," Journal of Pharmacology and Experimental Therapeutics, vol. 345, no. 2, pp. 206-214, 2013.

[131] S. E. Witta, R. M. Gemmill, F. R. Hirsch et al., "Restoring Ecadherin expression increases sensitivity to epidermal growth factor receptor inhibitors in lung cancer cell lines," Cancer Research, vol. 66, no. 2, pp. 944-950, 2006.

[132] M. Dohadwala, S. Yang, J. Luo et al., "Cyclooxygenase2-dependent regulation of E-cadherin: prostaglandin $\mathrm{E}(2)$ induces transcriptional repressors ZEB1 and snail in non-small cell lung cancer," Cancer Research, vol. 66, no. 10, pp. 5338-5345, 2006. 

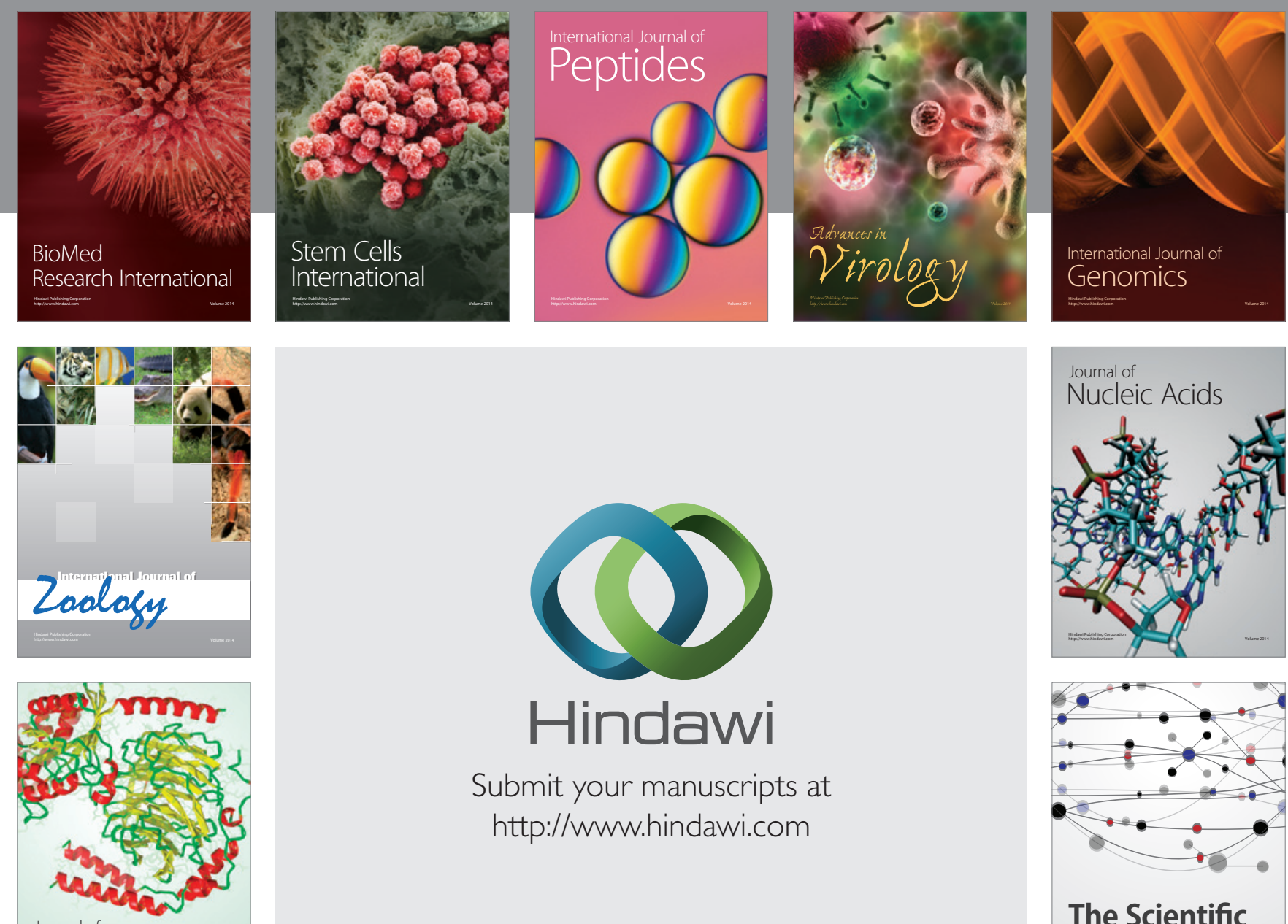

Submit your manuscripts at

http://www.hindawi.com

Journal of
Signal Transduction
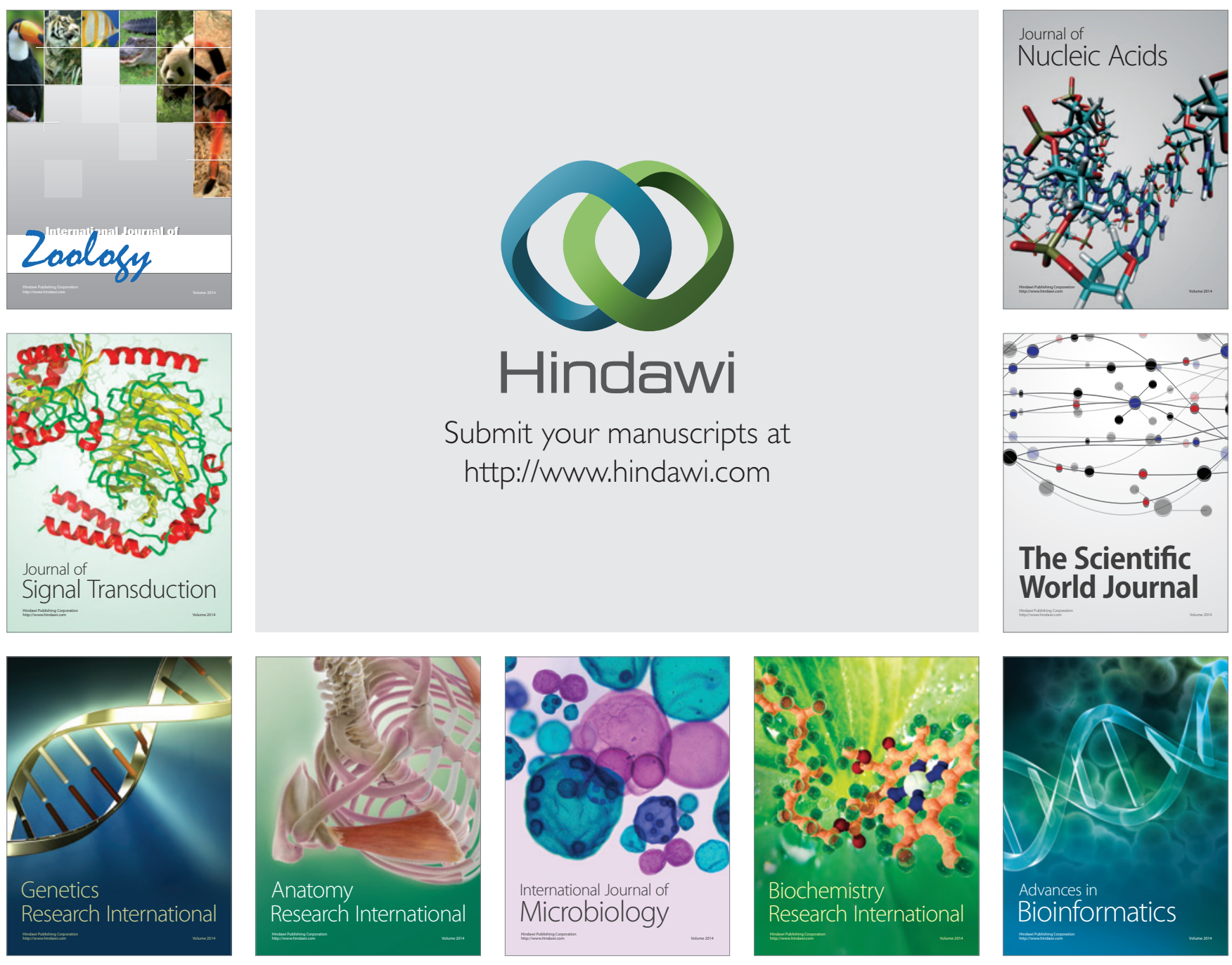

The Scientific World Journal
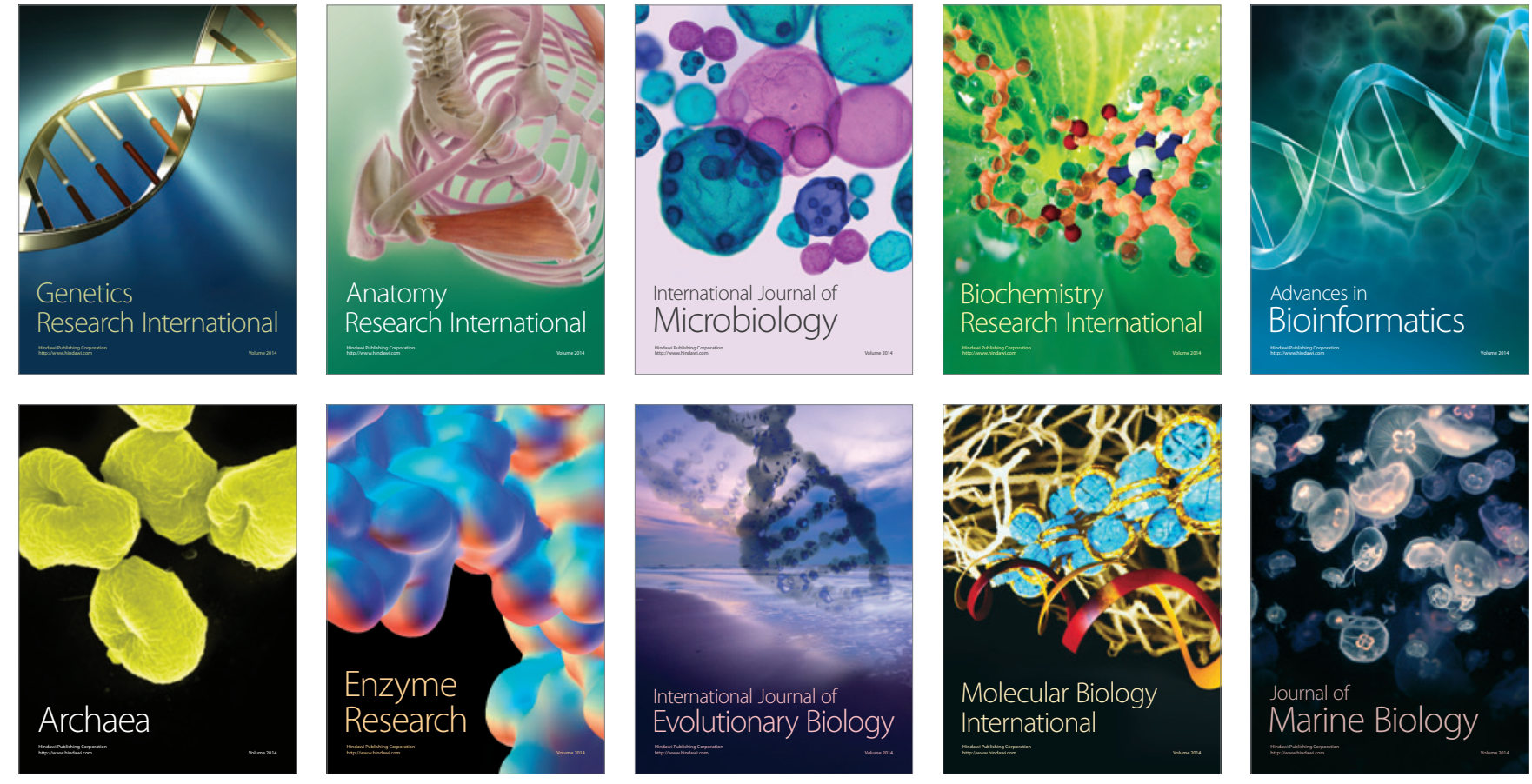